\title{
Estado del Arte de los Vehículos Autónomos Sumergibles Alimentados por Energía Solar
}

\author{
César Hernando Valencia Niño \\ MSc. da Engenharia Mecânica, \\ Universidade Federal do Rio de Janeiro \\ Docente Tiempo Completo, Director Línea de Investigación en \\ Automatización, Control y Robótica \\ Grupo de Aplicaciones Mecatrónicas - GRAM, \\ Universidad Santo Tomás \\ Bucaramanga, Colombia \\ cesar.valencia@ufrj.br
}

\author{
Max Suell Dutra \\ Dr.-Ing. Mecânica, \\ Gerhard Mercator Universität Gh Duisburg \\ Professor Associado COPPE, \\ Diretor Grupo de Pesquisa em Projetos de Maquinas e Robótica, \\ Universidade Federal do Rio de Janeiro \\ Rio de Janeiro, Brasil \\ max@mecanica.coppe.ufrj.br
}

\begin{abstract}
Resumen- En el presente artículo es desarrollado el estado del arte sobre los vehículos sumergibles y la evolución de los mismos desde 1945 hasta la fecha, de tal forma se hace una descripción de la evolución en todos los aspectos de diseño y alimentación desde los vehículos tripulados sin propulsión propia, se pasa por los vehículos con torpedos capaces de transportar una persona, se presentan los primeros submarinos de pequeño porte que conseguían velocidades altas para la época, después son presentados los vehículos remotamente operados con posibilidades de manipular objetos y obtener imágenes y finalmente los vehículos autónomos con elementos adicionales como el sistema de planeamiento de rutas y sistemas de navegación inercial para luego llegar a una variante de los mismos que contempla la posibilidad de utilizar energías alternativas como fuente de alimentación en este caso la energía solar.
\end{abstract}

Palabras clave— Inspección Submarina, Vehículos Remotamente Operados (ROV's), Vehículos Autónomos Sumergibles (AUV's) y Vehículos Autónomos Sumergibles Alimentados por Energía Solar (SAUV's).

\begin{abstract}
This article presents the state of the art of underwater vehicles and their evolution from 1945 to date. A description of developments in all aspects of design and energy source, starting with externally propelled and unmanned vehicles to the torpedo-capable vehicles that can carry a person is presented. The first small-sized high speed submarines for their time are described then. After these, all remotely operated vehicles with the potential to manipulate objects and images are presented. The review continues with autonomous vehicles with additional elements such as, route planning, and inertial navigation systems. It finishes with a variation of the latest developments, which provide the possibility of using alternative energy as a power source such as, in this case, solar energy.
\end{abstract}

Keywords-Submarine Inspection, Remotely Operated Vehicle (ROV's), Autonomous Underwater Vehicle (AUV's) and Solar-powered Autonomous Underwater Vehicle (SAUV).

\section{INTRODUCCIÓN}

Los vehículos sumergibles de la actualidad tienen misiones más complejas que sus antecesores. Por esto, el desarrollo de nuevos equipos tales como: sensores distribuidos, sistemas de navegación de tipo autónomo, obtención de la energía de fuentes alternativas, comunicaciones para distancias mayores y sistemas de control y planeamiento de rutas son utilizados en este tipo de vehículos.

En la historia muchos vehículos sumergibles fueron desarrollados para realizar todas estas tareas, los primeros conocidos como vehículos remotamente operados (Remote Operated Vehicles - ROV's), aún son utilizados con éxito, pero tienen restricciones para operaciones de mayor cobertura. Al crear nuevos niveles de trabajo son propuestos los vehículos autónomos sumergibles (Autonomous Underwater Vehicles - AUV's) según [1], los cuales presentan la ventaja de mayor capacidad de navegación con respecto a sus predecesores; en ellos los sistemas de planeamiento de rutas y control permiten aumentar las características de navegación de forma autónoma.

Para atender los requisitos de trabajo y aumentar la autonomía de operación sin intervención del personal de mantenimiento, se hace necesario optar por nuevas fuentes alternativas de energía. Así son consideradas aquellas que permiten el funcionamiento del vehículo y no disminuyen la capacidad de operación, para esto son utilizados equipos como pilas de combustible y celdas solares entre otros. 


\section{ESTADO DEL ARTE}

El constante interés del hombre por conocer los ambientes que existen en el planeta, pero que difícilmente son accesibles por las condiciones impuestas por el ambiente y por la naturaleza del cuerpo humano, genera una investigación que cobija muchas áreas de la ingeniería con el fin de desarrollar equipos que consigan satisfacer este interés. El estudio del fondo marino es un ejemplo de esta problemática, los resultados obtenidos en los inicios da la exploración presentan muchas dificultades de carácter tecnológico.

\section{A. Antecedentes Generales}

La primera problemática a ser resuelta fue la incapacidad de observar con claridad los objetos en el fondo marino, pues las imágenes que se presentan son confusas para el observador. Entonces la primera tarea fue crear una máscara que al interponer aire entre los ojos y el medio marino permitiese ver con claridad. Además de esto, fueron creadas las paletas contorneadas para permitir el desplazamiento con relativa facilidad, pero, una vez más los recursos fueron limitados como, por ejemplo, el aire que tiene el buzo no era suficiente para cubrir largas distancias.

Con el objetivo de aumentar la velocidad de desplazamiento y ofrecer comodidad para el buzo fue desarrollada la tabla de exploración submarina o vuelo submarino que es presentada en la Figura 1 y es el equipo más simple para desplazarse, el sistema está compuesto por una tabla que se mantiene sujeta a un barco a través de un cable de aproximadamente 5 metros, en ella están fijos los equipos como la brújula, el batímetro y el reloj como panel de instrumentos.

FIG. 1. TABLA DE EXPLORACIÓN SUBMARINA [2]
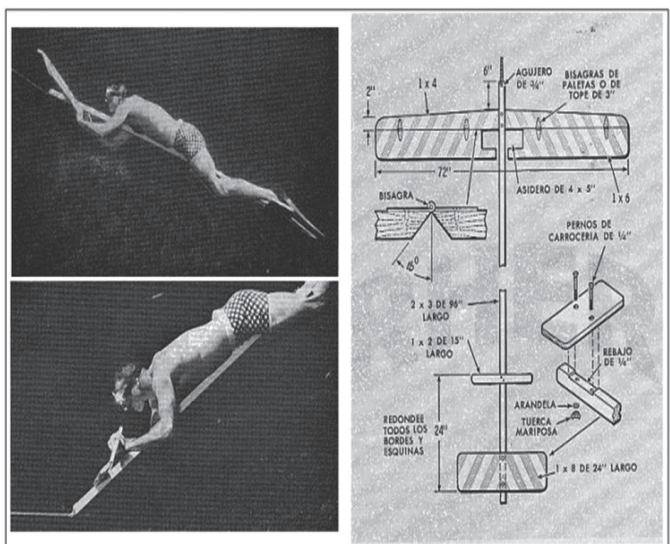

Este pequeño modelo fue rediseñado para convertirse en el Ala Volante de Vanlaer, que tenía la forma de un triángulo y que permitía toda clase de movimientos que el operador controlaba con una palanca. El siguiente paso fue desarrollar el propulsor sumergible, que constaba de un cilindro con un mecanismo de una hélice en el extremo posterior, uno de los primeros grupos de investigación que trabajó en esta evolución fue el Grupo de Estudios e Investigaciones Marinas de Francia en 1945.

En 1954 Dimitri Rebikoff construyó un torpedo sumergible con equipos de fotografía y filmación como es presentado en la Figura 2.

FIG. 2. TORPEDO SUMERGIBLE

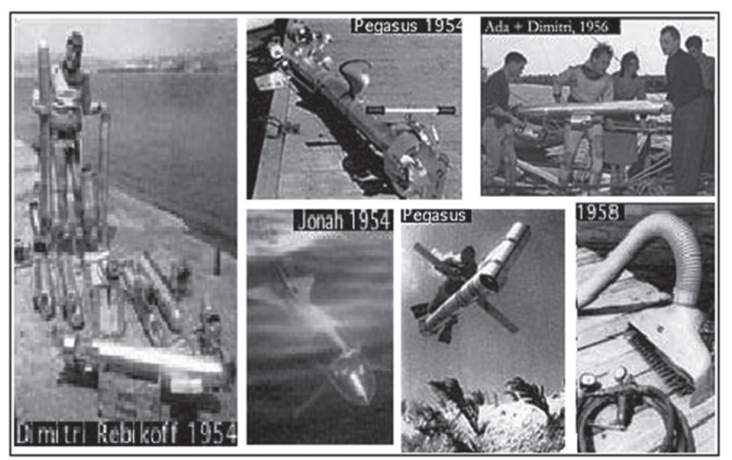

Fuente: Tomada de http://www.rebikoff.org

Pero la historia de la exploración submarina ha tenido muchos personajes, por ejemplo, el submarino Pino hecho en Italia, el sumergible Aluminaut que consiguió llegar a velocidades de $4,5 \mathrm{~km} / \mathrm{h}$, el Seapup, los Star-I, Star-Il y Star-III representan los últimos modelos desarrollados para los años $70^{\prime}$ s, en las Figuras 3 y 4 son presentados los modelos Aluminaut y Star-III respectivamente.

FIG. 3. SUBMARINO ALUMINAUT

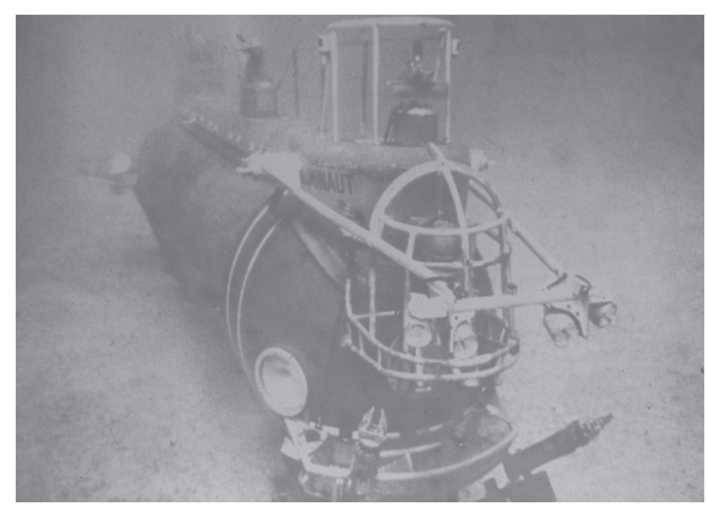

Fuente: Tomada de http://www.rebikoff.org 


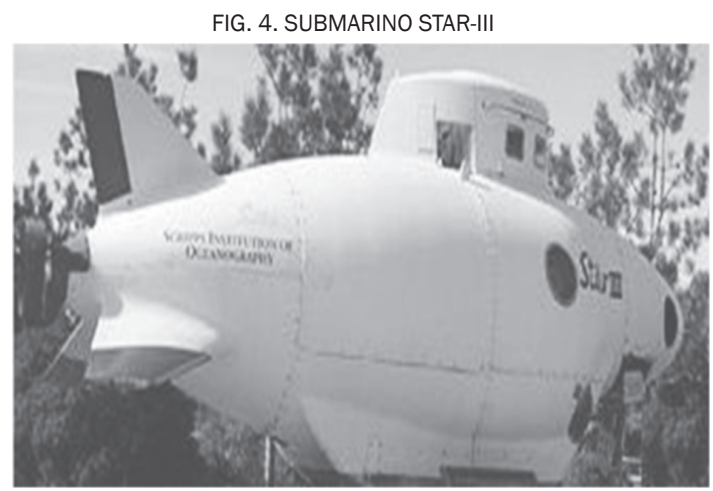

Fuente: Tomada de http://www.rebikoff.org

La exploración submarina evolucionó rápidamente para comienzos de los 80 con la utilización del primer vehículo sumergible operado remotamente en una plataforma de perforación de Noruega por la empresa i-Tech que es una subdivisión de la gigantesca Subsea 7. Pero la historia cambio para comienzos de los años 90 's cuando la Kongsberg Maritime desarrollo el primer vehículo autónomo sumergible (AUV), el cual tenía elementos para navegación autónoma que sería la evolución más representativa en estos vehículos; hoy estos vehículos forman parte de los principales dispositivos para investigación submarina.

\section{EVOLUCIÓN DEL PROBLEMA}

Como se dijo, la evolución de la investigación marina tiene muchas herramientas que han sido desarrolladas para la consecución de objetivos específicos de acuerdo con las necesidades de cada problema, los vehículos remotamente operados, ROV's, fueron desarrollados al rededor de los 80 por una gran cantidad de empresas dedicadas a la exploración petrolífera, en la Figura 5 se presentan los ROV's Panter Plus utilizado por la empresa SUB-FIND de Canadá y el Hyball de la empresa Inglesa HYBALL OFFSHORE.

FIG. 5. EJEMPLOS DE ROV`S [3]

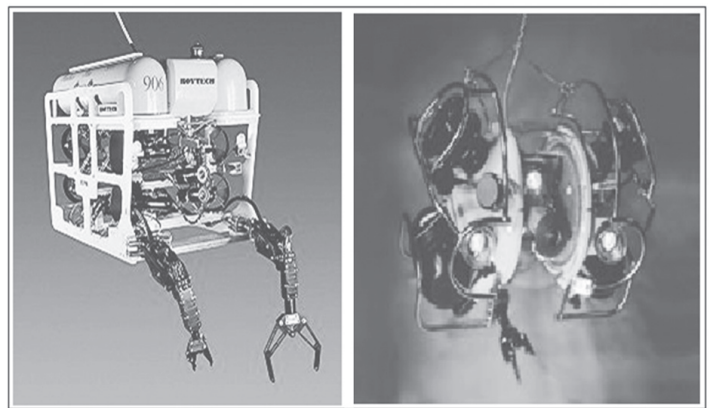

Actualmente, los ROV's son clasificados en cinco grandes grupos de acuerdo con las tareas que desempeñan:

Clase 1: vehículos solamente de observación;

Clase 2: vehículos para observación y transporte de pequeñas cargas;

Clase 3: vehículos para trabajos generales a nivel de intervención;

Clase 4: Tractores sumergibles o enterradores de líneas o cables; y

Clase 5: Prototipos y vehículos en desarrollo.

El desempeño de estos vehículos permitió una mayor autonomía de operación, teóricamente, para aquellos que son alimentados por un cordón umbilical, puesto que las baterías están en recarga constante en la superficie, para aquellos que tienen las baterías a bordo la autonomía de operación es reducida.

Las profundidades en que pueden ser utilizados varían, según los materiales con que fueron diseñados y la longitud del cable. Sin embargo, un modelo en particular superó todas las capacidades de operación consideradas actualmente, el Víctor-6000 que es presentado en la Figura 6 construido por Ifremer (Instituto Francés para investigación y exploración del mar).

FIG. 6. VICTOR $6000[4$
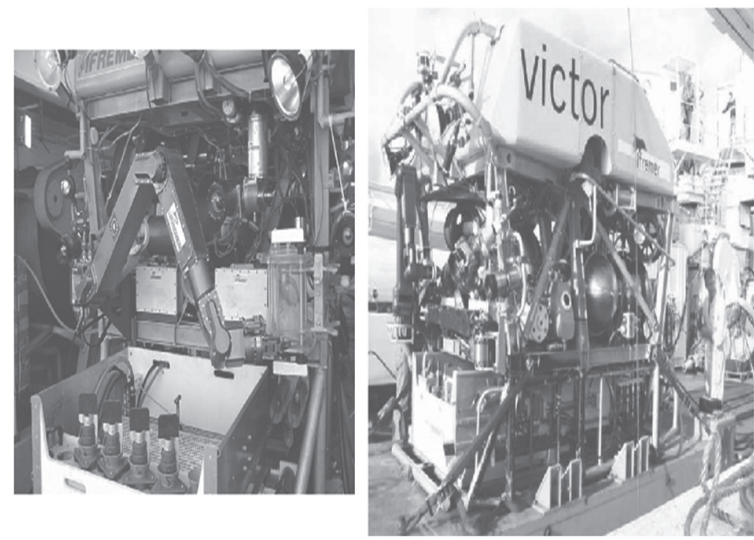

En tamaño es mayor que los ROV's utilizados para investigación comúnmente, sus dimensiones son de $3,1 \times 1,8 \times 2,1 \mathrm{~m}$ y su peso es de 4Ton, que podría ser considerado como un ROV para aplicaciones de gran porte, consigue velocidades de hasta 1,5nudos tiene un conjunto de 8 cámaras para adquisición de imágenes, pero la particularidad de este vehículo es conseguir 
una profundidad de $6 \mathrm{~km}$, sólo que para conseguir dicha profundidad el peso del cable es mayor que el de cualquier otro sistema utilizado antes, para esto, es utilizado el sistema de jaula presentado en la Figura 7.

FIG. 7. SISTEMA COMPLETO DE VICTOR-6000 [5]

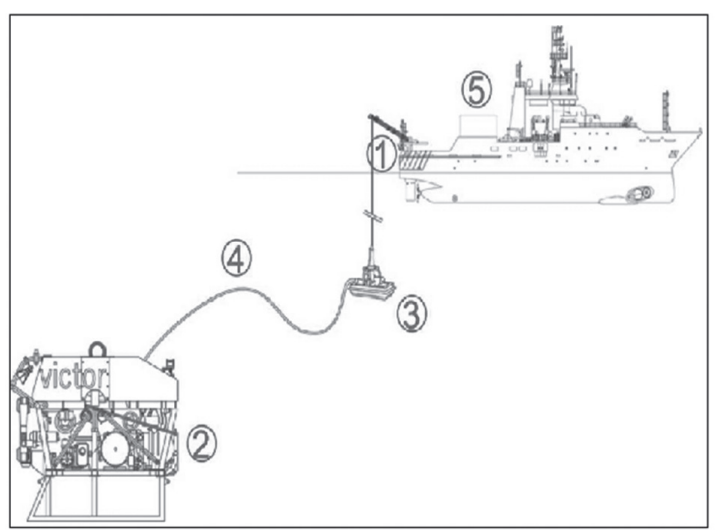

En la Tabla I son presentados los diferentes módulos que componen el sistema Victor-6000.

TABLA I

\begin{tabular}{|c|l|}
\multicolumn{2}{c}{} \\
\hline Referencia & \multicolumn{1}{c|}{ Módulos del sistema Victor-6000 [5] } \\
\hline 1 & $\begin{array}{l}\text { Grúa hidráulica con un cordón umbilical de } \\
\text { 20mm para una longitud total de 8500m } \\
\text { con capacidad para soportar 294kN. }\end{array}$ \\
\hline 2 & módulos de Inspección remota (ROV). \\
\hline 3 & $\begin{array}{l}\text { Lastre “Hard Ballast” de 1,5x0,8x0,5m con } \\
\text { un peso de 11kN. }\end{array}$ \\
\hline 4 & $\begin{array}{l}\text { cordón umbilical de 35mm para longitudes } \\
\text { variables hasta 300m. }\end{array}$ \\
\hline 5 & $\begin{array}{l}\text { Barco en la superficie que tendrá la grúa y } \\
\text { los paneles de control del ROV. }\end{array}$ \\
\hline
\end{tabular}

El primer AUV fue el Hugin presentado en la Figura 8 desarrollado alrededor de los 90 en una asociación internacional compuesta por Kongsberg Maritime, Departamento de Investigaciones de Defensa de Noruega, Statoil, Nui As, C\&C Technologies, Geoconsult AS, BPy Norsk Hydro, mediante sistemas como el (Differential Global Positioning System - DGPS y Ultra Short Base Line - USBL) presentados en [6] y (NAVLAB) presentado en [7], el blanco de su construcción fueron aplicaciones civiles como investigación y control ambiental y aplicaciones militares como el conteo de minas en el mar.

Dichos vehículos tenían la capacidad de transportar diferentes tipos de sensores, sin embargo su envergadura era grande para los desarrollados hoy en día y necesitaban de medios de transporte grandes.

FIG. 8. VEHÍCULO HUGIN [8]

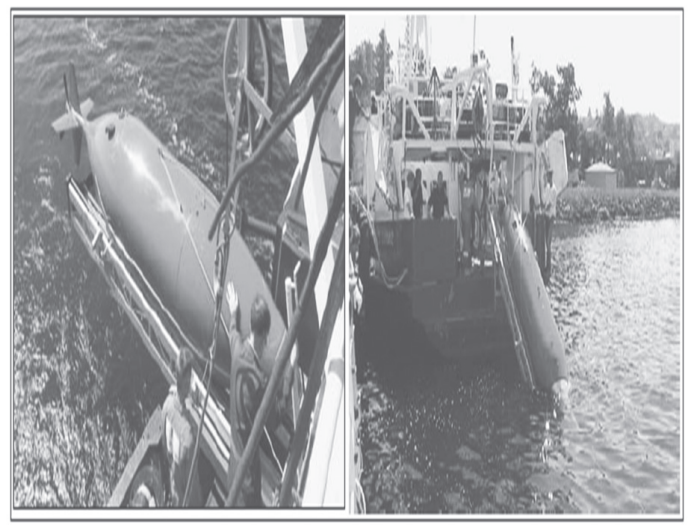

Las tareas realizadas por estos dispositivos son principalmente de inspección, investigación y recolección de muestras. En ellos se tiene una mayor autonomía de navegación debido al planeamiento de las trayectorias (Figura 9) donde son escogidas para atender puntos específicos basados en curvas batimétricas, pero, la autonomía estaba condicionada al rendimiento de las baterías de a bordo, para este tipo de vehículos se tienen dos tipos de navegación:

- Navegación autónoma sin referencia inercial; y

- Navegación autónoma con referencia inercial.

FIG. 9. TRAYECTORIAS DEL SISTEMA DE NAVEGACIÓN [9]

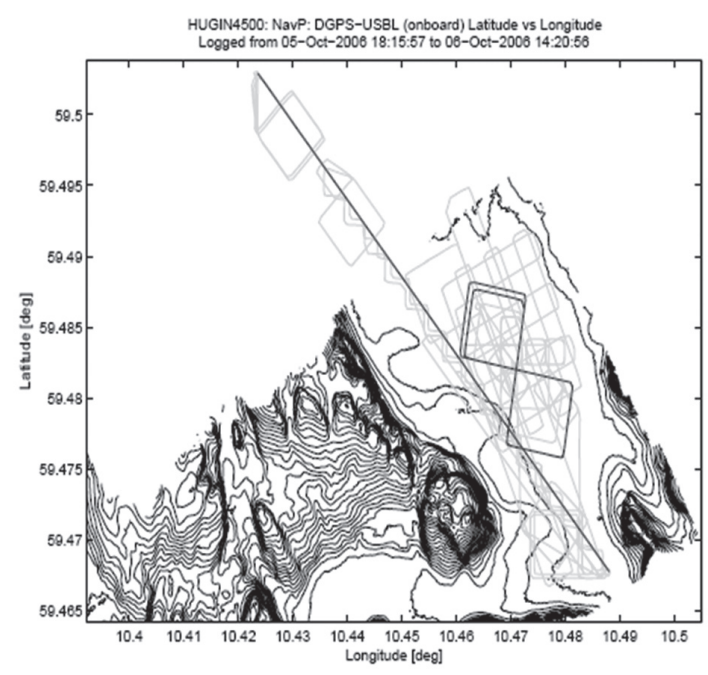

Cuando son utilizados sistemas sin referencia inercial estos tienen aplicaciones de tipo furtivo y son usados principalmente por las fuerzas militares, trabajan con sistemas de navegación 
que son orientados basándose en los movimientos del vehículo, los sistemas con referencia inercial necesitan tener sistemas auxiliares externos para hacer una constante comparación y disminuir el error, estos vehículos tienen los siguientes subsistemas:

- Sistema de Navegación;

- Sistema de Sensores;

- Sistema de Energía;

- Sistema de Control; y

- Sistema de Comunicaciones.

En los subsistemas de sensores y comunicaciones los AUV's no tienen diferencia con los $R O V ' s$, en el subsistema de energía la mayor diferencia es con aquellos vehículos que son alimentados por cordones umbilicales.

Los sistemas de control y navegación según [10] fueron rediseñados para tomar decisiones acertadas que mantengan las trayectorias que fueron planeadas por el control de misión.

FIG. 10. SISTEMA DE NAVEGACIÓN CON USBL [10]

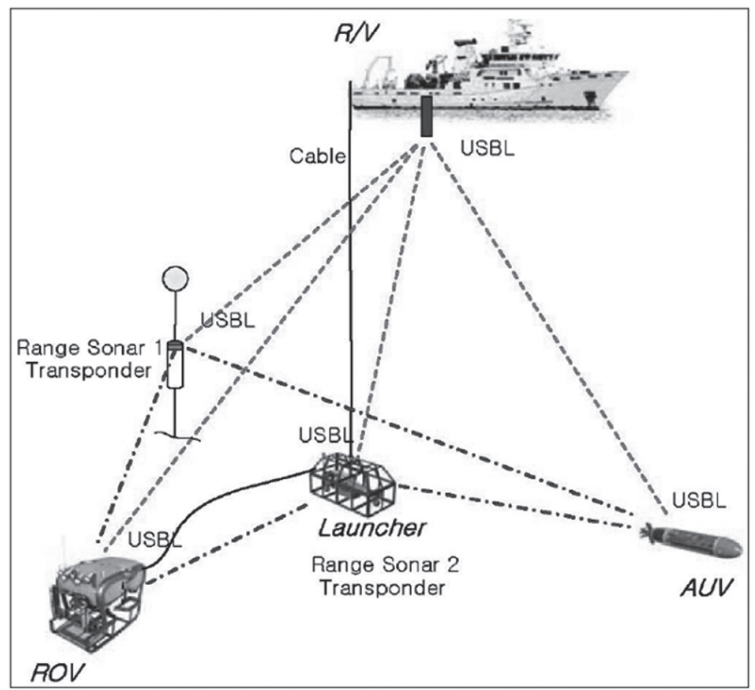

Actualmente estos vehículos son utilizados de forma constante en los sistemas offshore como equipo de apoyo e inspección(Figura 10). Estos sistemas utilizan dispositivos de caracterización por medio de ultra-sonido tienen la capacidad de obtener el mapeo de la superficie marina y de los objetos existentes en el fondo del mar, como por ejemplo, tuberías submarinas y posibles escapes o fugas (Figura 11).
FIG. 11. MAPEO CON EL AUV GAVEA [11]

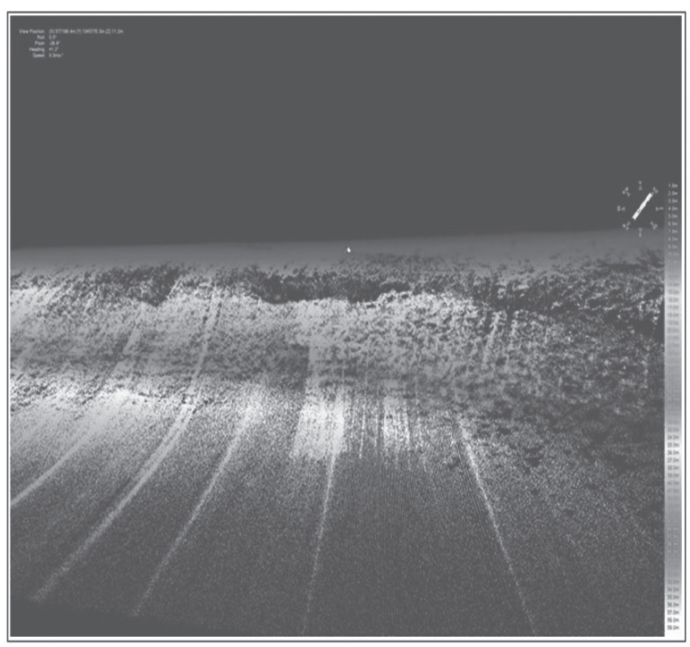

Con los avances de la tecnología y la concepción de las fuentes alternativas de energía, se han propuesto modelos factibles de ser implementados y que pueden sustituir los existentes para mejorar las características de desempeño.

En 1997 el programa cooperativo de investigación comenzó con el Instituto de Sistemas Autónomos Submarinos(Autonomous Undersea Systems Institute - AUSI), Instituto en tecnología Marítima (Institute for Maritime Technology - IMT) y la Academia Rusa de Ciencias (Russian Academy of Science - RAS) que originó el primer vehículo autónomo sumergible alimentado por energía solar (Solar-Powered Autonomous Underwater Vehicle - SAUV). Con esta cooperación el AUSI se tornó como el mayor instituto de investigación en esta área en el mundo, con experimentos desarrollados cuya duración es hasta de 30 días $[12,13]$. Los resultados obtenidos permiten verificar las ventajas del uso de paneles solares como fuente de energía. (Figura 12).

FIG. 12. RESULTADOS DE LOS PANELES SOLARES [14]

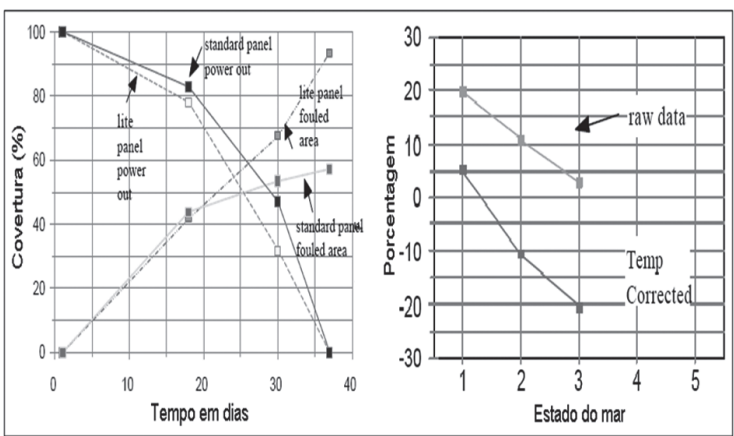


Los investigadores del AUSI son los inventores del protocolo AUSNET $[15,16,17]$ utilizado para comunicaciones y desarrollaron el concepto de sistemas cooperativos en AUV's según $[18,19]$, además, utiliza el enrutado dinámico y el sistema cooperativo AUV Development Concept CADCON [20](Figura 13).

FIG. 13. SISTEMAS COOPERATIVOS [20]

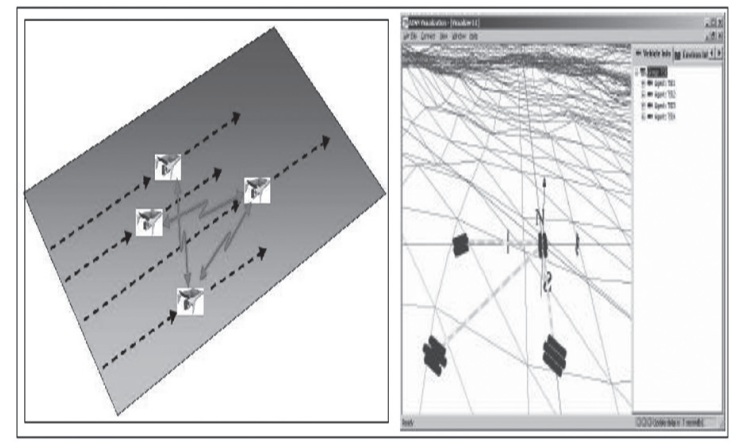

El modelo ha cambiado en la estructura $[21,22,23,24]$, ahora es necesario incluir los paneles solares(Figura 14) en el vehículo de tal forma que cuando este en la superficie pueda adquirir la mayor cantidad de sol posible para cargar las baterías de a bordo.

Este proceso es autónomo y repetitivo por largos periodos de tiempo para así generar la auto-suficiencia necesaria para realizar tareas sin la intervención del equipo de mantenimiento a pesar de los inconvenientes generados por las olas de mar según [25].

FIG. 14. SAUV-II DESARROLLADO POR AUSI. [23]

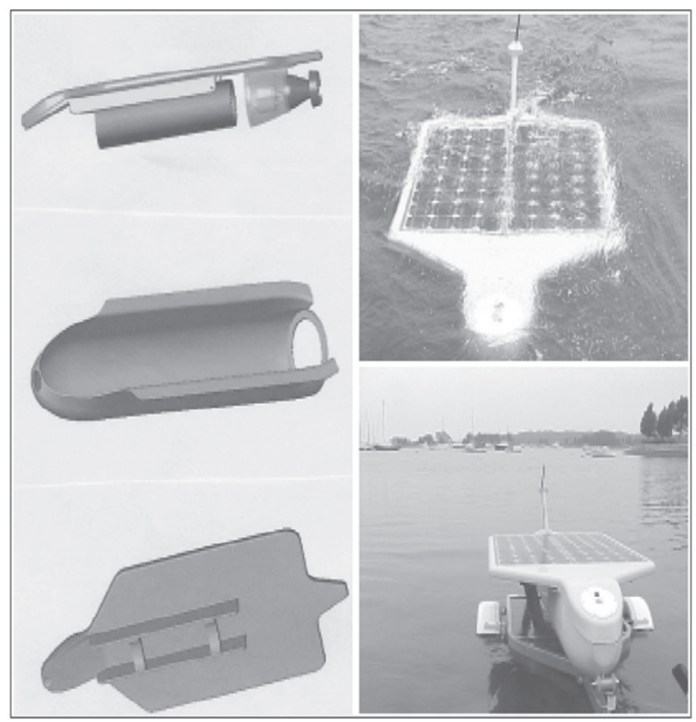

\section{CENTROS DE INVESTIGACIÓN}

En este momento alrededor del mundo hay una cantidad considerable de centros de desarrollo e investigación en el diseño y construcción de este tipo de vehículos, además de tener simposios, conferencias y seminarios como:

- International Symposium on Underwater Technology;

- International Symposium on Unmanned Untethered Submersible Technology;

- OCEANS Conference MTS/IEEE;

- Workshop on Autonomous Underwater Vehicles; $y$

- Workshop on Scientific Use of Submarine Cables and Related Technologies.

En la Tabla II son presentados los principales centros de investigación en el mundo. Es posible observar que la investigación en esta área tiene bastante competencia y la utilización de este tipo de vehículos está en el foco de empresas, centros de investigación, universidades e instituciones independientes.

TABLA II

Principales centros de investigación en el mundo

\begin{tabular}{|c|c|c|}
\hline Dependencia & Institución/Sede & País \\
\hline $\begin{array}{c}\text { Australian } \\
\text { Centre for Fields } \\
\text { Robotics }\end{array}$ & Sydney & Australia \\
\hline $\begin{array}{l}\text { Autonomous } \\
\text { System Labo- } \\
\text { ratory }\end{array}$ & $\begin{array}{c}\text { University of Hawaii } \\
\text { at Manoa }\end{array}$ & USA \\
\hline $\begin{array}{l}\text { Autonomous Un- } \\
\text { dersea System } \\
\text { Institute }\end{array}$ & New Hampshire & USA \\
\hline $\begin{array}{c}\text { Autonomous } \\
\text { Underwater } \\
\text { Vehicles Group }\end{array}$ & Uppsala University & Suiza \\
\hline $\begin{array}{l}\text { Center of Ocean } \\
\text { Engineering }\end{array}$ & $\begin{array}{l}\text { Massachusetts Insti- } \\
\text { tute Technology }\end{array}$ & USA \\
\hline $\begin{array}{l}\text { Centro de Eng- } \\
\text { enharia e Tecno- } \\
\text { logia Naval }\end{array}$ & $\begin{array}{c}\text { Universidade Técnica } \\
\text { de Lisboa }\end{array}$ & Portugal \\
\hline $\begin{array}{c}\text { Computer Vision } \\
\text { Research Labo- } \\
\text { ratory }\end{array}$ & $\begin{array}{l}\text { University of Massa- } \\
\text { chusetts }\end{array}$ & USA \\
\hline $\begin{array}{c}\text { Control \& } \\
\text { Instrumentation } \\
\text { Systems Group }\end{array}$ & University of Limerick & Irlanda \\
\hline
\end{tabular}




\begin{tabular}{|c|c|c|}
\hline DeepC & STN - Atlas Elektronik & Alemania \\
\hline $\begin{array}{l}\text { Deepsea Re- } \\
\text { search }\end{array}$ & $\begin{array}{l}\text { Alfred Wegener Insti- } \\
\text { tute (AWI) }\end{array}$ & Alemania \\
\hline $\begin{array}{l}\text { Deep Submer- } \\
\text { gence Labora- } \\
\text { tory }\end{array}$ & $\begin{array}{l}\text { Woods Hole Oceano- } \\
\text { graphic Institution }\end{array}$ & USA \\
\hline $\begin{array}{l}\text { Department of } \\
\text { Automation }\end{array}$ & $\begin{array}{l}\text { Technical University } \\
\text { of Denmark }\end{array}$ & Dinamarca \\
\hline $\begin{array}{l}\text { Dynamic Sys- } \\
\text { tems and Oce- } \\
\text { anic Robotics }\end{array}$ & $\begin{array}{c}\text { Universidade Técnica } \\
\text { de Lisboa }\end{array}$ & Portugal \\
\hline $\begin{array}{l}\text { French Re- } \\
\text { search Institute } \\
\text { for Exploitation } \\
\text { of the Sea }\end{array}$ & Paris & Francia \\
\hline $\begin{array}{l}\text { Grupo de Visión } \\
\text { por Computador } \\
\text { y Robótica }\end{array}$ & $\begin{array}{l}\text { Universidad de } \\
\text { Girona }\end{array}$ & España \\
\hline $\begin{array}{l}\text { Grupo de Aplica- } \\
\text { ciones Mecatró- } \\
\text { nicas GRAM }\end{array}$ & $\begin{array}{l}\text { Universidad Santo } \\
\text { Tomás } \\
\text { Seccional Bucara- } \\
\text { manga }\end{array}$ & Colombia \\
\hline $\begin{array}{l}\text { Institute for Ma- } \\
\text { rine Dynamics }\end{array}$ & $\begin{array}{l}\text { National Research } \\
\text { Council (NRC) of } \\
\text { Canada }\end{array}$ & Canadá \\
\hline $\begin{array}{c}\text { Institute of } \\
\text { Oceanography }\end{array}$ & $\begin{array}{l}\text { Hellenic Centre for } \\
\text { Marine Research }\end{array}$ & Grecia \\
\hline $\begin{array}{l}\text { Kato (Un- } \\
\text { derwater Robo- } \\
\text { tics) Lab. }\end{array}$ & Tokai University & Japón \\
\hline $\begin{array}{l}\text { Laboratório } \\
\text { de Projeto de } \\
\text { Maquinas e } \\
\text { Robótica }\end{array}$ & $\begin{array}{l}\text { Universidade Federal } \\
\text { do Rio de Janeiro }\end{array}$ & Brasil \\
\hline $\begin{array}{l}\text { Marine Techno- } \\
\text { logy Department }\end{array}$ & JAMSTEC & Japón \\
\hline $\begin{array}{c}\text { Mechatronics } \\
\text { Research Centre }\end{array}$ & $\begin{array}{l}\text { University of Wales } \\
\text { College }\end{array}$ & Inglaterra \\
\hline $\begin{array}{l}\text { Motion Control } \\
\text { Research Group }\end{array}$ & $\begin{array}{l}\text { Norwegian Univer- } \\
\text { sity of Science and } \\
\text { Technology }\end{array}$ & Noruega \\
\hline $\begin{array}{c}\text { Ocean Systems } \\
\text { Laboratory }\end{array}$ & $\begin{array}{l}\text { Heriot-Watt Univer- } \\
\text { sity }\end{array}$ & Inglaterra \\
\hline $\begin{array}{l}\text { P.P. Shirshov } \\
\text { Institute of } \\
\text { Oceanology }\end{array}$ & $\begin{array}{l}\text { Russian Academy of } \\
\text { Sciences }\end{array}$ & Rusia \\
\hline $\begin{array}{l}\text { Robotic Labo- } \\
\text { ratory }\end{array}$ & $\begin{array}{c}\text { Intelligent Systems } \\
\text { for Automation } \\
\text { Institute }\end{array}$ & Italia \\
\hline
\end{tabular}

En la consulta de las diferentes fuentes bibliográficas, laboratorios y centros de investigación fue confirmado que la utilización de los vehículos autónomos para tareas de inspección, monitoriamiento, recolección de muestras y otras, es de gran importancia. Estos vehículos se han convertido en un apoyo para misiones donde las condiciones del ambiente no permiten al hombre llegar o donde sólo se puede permanecer durante poco tiempo. Diversos trabajos de investigación y desarrollo han sido realizados en esta área del conocimiento en centros de investigaciones del mundo y este articulo es una contribución inicial del Grupo de Investigación en Diseño de Máquinas y Robótica (LabRob) de la UFRJ y el grupo de aplicaciones mecatrónicas (GRAM) de la USTA seccional Bucaramanga.

\section{Agradecimientos}

Los autores agradecen la contribución del Ing. Jorge Guillermo Díaz por la asesoría en el resumen del presente artículo.

\section{REFERENCIAS}

[1] C. H. VALENCIA, "Estudo de um veículo autônomo submersível alimentado por energia solar," Tesis de Maestría, Universidad Federal do Rio de Janeiro, Programa de Ingeniería Mecánica, Cap. 1, Brasil, 2009

[2] W. MORRIS, “Acuaplano Submarino," Revista Mecánica Popular, v. 20, pp. 50-51, 1967

[3] NORSOK STANDARD, "Remotely Operated Vehicle (ROV) Services," U-102, Rev. 1, October 2003

[4] J-L. MICHEL, M. KLAGES, F. J. BARRIGA, et al, "Victor 6000: Design, Utilization and First Improvements," Thirteenth International Offshore and Polar Engineering Conference, ISBN 1-880653-60-5, Honolulu, Hawaii, USA, 25-30 May 2003

[5] T. SOLTWEDEL, M. KLAGES, "First Deployment of the French ROV VICTOR 6000 from board the German Research Icebreaker RV POLARSTERN," Sea Technology, v. 41, no. 4, pp. 51-53, April. 2000

[6] M. MANDT, K. GADE, B. JALVING, "Integrating DGPSUSBL Position Measurement with Inertial Navigation in the HUGIN 3000 AUV," 8th Saint Petersburg International Conference on Integrated Navigation Systems, pp. 63-74, Saint Petersburg, Russia, May. 2001

[7] K. GADE, “NavLab, a Generic Simulation and Post-Processing Tool Navigation," European Journal of Navigation, v. 2, no. 4, pp. 1-9, November. 2004

[8] R. MARTHINIUSSEN, K. VESTGARD, R. A. KLEPAKER, et al, "Hugin-AUV Concept and Operational Experiences 
to Date," OCEANS 2004 Conference MTS/IEEE, v. 2 pp. 846-850, Kobe, Japan, November 2004

[9] O. HEGRENSES, O. HALLINGSTAD, B. JALVING, "Comparison of Mathematical Models for the HUGIN 4500 AUV Based on Experimental Data," Symposium on Underwater Technology and Workshop on Scientific Use of Submarine Cables and Related Technologies, pp. 558-567, Tokyo, Japan, April. 2007

[10] L. PAN-MOOK, J. BONQ-HUAN, T. C. HYUN, et al, "An Integrated Navigation System for Underwater Vehicles Based on Inertial Sensors and Pseudo LBL Acoustic Transponders," OCEANS 2005 Conference MTS/IEEE, pp. 555-562, Brest, Finistere, France, June. 2005

[11] A. TREMBANIS, "As The Gigabytes Of Data Come Streaming In!. In: Bonaire 2008: Exploring Coral Reef Sustainability With New Technologies," NOAA Ocean Explorer, Silver Spring, Maryland, USA, 2008

[12] D. CRIMMINS, C. DEACUTIS, E. HINCHEY, et al, "Use of a Long Endurance Solar Powered Autonomous Underwater Vehicle (SAUV II) to Measure Dissolved Oxygen Concentration in Greenwich Bay, Rhode Island, U.S.A," OCEANS 2005 Conference MTS/IEEE, v. 2, pp. 896-901, Brest, Finistere, France, June. 2005

[13] D. M. CRIMMINS, C. T. PATTY, M. A. BELIARD, et al, "Long-Endurance Test Results of the Solar-Powered AUV System," OCEANS 2006 Conference MTS/IEEE, pp. 1-5, Boston, Massachusetts, USA, September. 2006

[14] M. D. AGEEV, D. R. BLIDBERG, J. JALBERT, et al, "Results of the Evaluation and Testing of the Solar Powered AUV and its Subsystems," Workshop on Autonomous Underwater Vehicles, pp. 137-145, San Antonio, Texas, USA, June. 2002

[15] M. HAAG, E. AGU, R. KOMERSKA, et al, "Status packet deprecation and store-forward routing in AUSNet," International Conference on Mobile Computing an Networking, pp. 86-92, Los Angeles, California, USA, September. 2006

[16] C. BENTON, J. KENNEY, S. G. CHAPPELL, et al, "Autonomous undersea systems network (AUSNET) development status update," OCEANS 2002 MTS/IEEE, v. 1, pp. 325-330, Biloxi, Mississippi, USA, October. 2002.

[17] C. BENTON, J. KENNEY, R. NITZEL, et al, "Autonomous undersea systems network (AUSNet) - protocols to support ad-hoc UV communications," IEEE/OES Autonomous Underwater Vehicles, pp. 83-87, Sebasco ME, USA, June. 2004

[18] S.G. CHAPPEL, R. J. KOMERSKA, L. PENG, et al, "Cooperative AUV Development Concept (CADCON) An Environment for High-Level Multiple AUV Simulation," 11th International Symposium on Unmanned Untethered Submersible Technology, pp. 112-120, Durham, USA, August. 1999
[19] C. N. DUARTE, G. R. MARTEL, C. BUZZELL, et al, “A Common Control Language to Support Multiple Cooperating AUV's," 14th International Symposium on Unmanned Untethered Submersible Technology, Durham, USA, August. 2005

[20] R. NITZEL, C. BENTON, S. CHAPPELL, et al, “Exploiting dynamic source routing to enable undersea networking over an ad-hoc topology," International Symposium on Underwater Technology, pp. 273-277, Tokyo, Japan, April. 2002

[21] J. JALBERT, D. BLIDBERG, M. AGEEV, "Some design considerations for a solar-powered AUV: Energy management and its impact on operational characteristics," Unmanned Undersea Systems, pp. 26-31, 1997

[22] R. BLIDBERG, J. JALBERT, M. D. AGEEV, "The AUSI/ IMTP solar powered autonomous undersea vehicle," OCEANS 1998, v. 1, pp. 363-368, Nice, France, October. 1998

[23] J. JALBERT, J. BAKER, J. DUCHESNEY, et al, "A solarpowered autonomous underwater vehicle," OCEANS 2003 MTS/IEEE, v. 2, pp. 1132-1140, San Diego, California, USA, September. 2003

[24] C. H. VALENCIA, M. S. DUTRA, R. RAMIREZ, "Solar Powered Autonomous Underwater Vehicle Subsystems", México, Electronics, Robotics and Automotive Mechanics Conference - CERMA2008, ISBN: 978-07695-3320-9

[25] C. H. VALENCIA, M. S. DUTRA, R. RAMIREZ, et al, "Predição da Eficiência de Recarga de Baterias de um SAUV por Efeito das Ondas do Mar", V Congresso Nacional de Engenharia Mecânica - CONEM2008, pp. $328-329$ 